\title{
Condições que interferem no cuidado às mulheres em situação de violência conjugal
}

\author{
Conditions that interfere in the care of women in situation of conjugal violence
}

Condiciones que interfieren en la atención de mujeres en situación de violencia conjugal

\author{
Jordana Brock Carneiro ${ }^{1}$ (D) \\ Nadirlene Pereira Gomes ${ }^{1}$ (b) \\ Lilian Conceição Guimarães de Almeida ${ }^{1}$ (1) \\ Cátia Maria Costa Romano ${ }^{1}$ (D) \\ Andrey Ferreira da Silva ${ }^{1}$ (i) \\ Natália Webler ${ }^{1}$ (b)
}

Maria Deolinda Antunes Luz Lopes Dias

Mauricio $^{2}$ (1)

1. Universidade Federal da Bahia, Escola de

Enfermagem. Salvador, BA, Brasil.

2. Escola Superior de Enfermagem de Lisboa. Lisboa, Portugal.
Autor correspondente:

Jordana Brock Carneiro

E-mail: jordanabrock@yahoo.com.br

Recebido em 17/03/2021.

Aprovado em 12/07/2021.

\section{Resumo}

Objetivo: conhecer as condições que interferem no cuidado às mulheres em situação de violência conjugal. Métodos: estudo qualitativo ancorado na Teoria Fundamentada nos Dados. Foram realizadas entrevistas, entre fevereiro e dezembro de 2019 , com 31 profissionais de saúde atuantes em Unidades de Saúde da Família de um município do Nordeste brasileiro, as quais integraram um dos dois grupos amostrais (profissionais da Estratégia de Saúde da Família e Núcleo de Apoio à Saúde da Família). Resultados: os elementos que interferem no cuidado à mulher em situação de violência conjugal foram representados nas categorias: Entendendo a importância da atuação profissional organizada; Reconhecendo a necessidade de preparo profissional para enfrentamento da violência conjugal; Percebendo a essencialidade do fluxo de atendimento intersetorial. Considerações finais e impactos para a prática: o estudo revelou que o cuidado à mulher em situação de violência conjugal perpassa pelo preparo profissional, pela organização dos serviços de saúde e um fluxo de atendimento articulado e intersetorial. Nesse sentido, oferece subsídios que podem orientar gestores para a elaboração ações de identificação e enfrentamento da violência conjugal contra a mulher, pautadas na coparticipação e corresponsabilização das trabalhadoras da Estratégia de Saúde da Família, com fins em melhorias na assistência ofertada.

Descritores: Violência contra a Mulher; Violência por Parceiro Íntimo; Enfermagem; Assistência à Saúde; Atenção Primária à Saúde.

\section{Abstract}

Objective: to know the conditions that interfere in the care of women in situation of marital violence. Methods: a qualitative study anchored in Grounded Theory. Interviews were conducted, between February and December 2019, with 31 health professionals working in Family Health Units of a municipality in Northeastern Brazil, which integrated one of the two sample groups (professionals of the Family Health Strategy and Family Health Support Center). Results: the elements that interfere in the care of women in situations of marital violence were represented in the categories: Understanding the importance of organized professiona action; Recognizing the need for professional preparation to deal with marital violence; Realizing the essentiality of the flow of intersectoral care. Final considerations and impacts for practice: the study revealed that the care for women in situations of marital violence goes through the professional preparation, the organization of health services and a flow of articulated and intersectoral care. In this sense, it offers subsidies that can guide managers to develop actions to identify and address marital violence against women, based on the co-participation and co-responsibility of the workers of the Family Health Strategy, in order to improve the assistance offered.

Descriptors: Domestic Violence; Intimate Partner Violence; Nursing; Delivery of Health Care; Primary Health Care.

\section{Resumen}

Objetivo: conocer las condiciones que interfieren en el cuidado de las mujeres en situaciones de violencia conyugal. Métodos: estudio cualitativo basado en la Teoría Fundamentada en los Datos. Las entrevistas se realizaron entre febrero y diciembre de 2019 con 31 profesionales de la salud que laboran en Unidades de Salud de la Familia en un municipio del noreste de Brasil, quienes formaron parte de uno de los dos grupos de la muestra (profesionales del Centro de Estrategia de Salud de la Familia y Apoyo a la Salud de la Familia). Resultados: Ios elementos que interfieren en el cuidado de la mujer en situación de violencia intrafamiliar fueron representados en las categorías: Comprensión de la importancia de la práctica profesional organizada; Reconociendo la necesidad de preparación profesional para enfrentar la violencia doméstica; Darse cuenta de la esencialidad del flujo de atención intersectorial. Consideraciones finales e impactos para la práctica: el estudio reveló que la atención a la mujer en situación de violencia conyugal permea la preparación profesional, la organización de los servicios de salud y un flujo de atención articulado e intersectorial. En este sentido, ofrece subsidios que pueden orientar a los gestores a desarrollar acciones de identificación y enfrentamiento de la violencia conyugal contra las mujeres, basadas en la coparticipación y corresponsabilidad de los trabajadores en la Estrategia de Salud de la Familia, con el objetivo de mejorar la atención brindada. 


\section{INTRODUÇÃO}

A violência por parceiro íntimo, também denominada violência conjugal, configura-se como um problema mundial de saúde pública, tendo em vista a sua alta incidência na sociedade e suas repercussões para a vida e saúde de mulheres ${ }^{1}$. Apesar dos consideráveis avanços nas políticas públicas de enfrentamento a esse agravo, nota-se que as estatísticas permanecem alarmantes, o que sinaliza para a fragilidade do cuidado ofertado à mulher, sobretudo, na Rede de Enfrentamento à Violência Contra a Mulher, onde se insere a Atenção Primária à Saúde (APS $)^{2,3}$.

Evidenciando a ampla dimensão do problema, dados da Organização das Nações Unidas revelam que, no ano de 2017 , cerca de $17,8 \%$ das mulheres no mundo foram vítimas de violências física ou sexual cometidas pelos companheiros nos últimos 12 meses ${ }^{1}$. No Brasil, levantamento de abrangência nacional também aponta para dados preocupantes ao elucidar a ocorrência de 263.067 casos de violência doméstica somente no ano de $2018^{4}$. Observa-se que os dados desse agravo no País permanecem alarmantes, visto que, apenas durante os cinco primeiros meses de 2021, já foram registradas, através das centrais telefônicas Disque 100 e/ou Ligue 180, 25.331 denúncias de violência por mulheres contra seus parceiros ou ex-parceiros íntimos, sendo uma média de aproximadamente 169 ligações por dia 5 .

Esse contexto tem suscetibilizado as mulheres ao adoecimento físico e psicológico, além de causar graves comprometimentos nas interações sociais. Dentre as manifestações clínicas destacam-se as escoriações, fraturas, queimaduras, lacerações vaginais, infecções sexualmente transmissíveis (IST's), gravidezes indesejadas, processos de abortamento, além do acometimento psíquico evidenciado, entre outras formas, pelas doenças psicossomáticas, depressão e comportamento suicida ${ }^{6,7}$. Todo esse processo pode fazer com que as mulheres tendam a se afastar do convívio social, o que dificulta a ruptura do ciclo da violência ${ }^{6}$.

Diante da magnitude desse fenômeno, percebeu-se a necessidade do estabelecimento de uma Rede de Enfrentamento à Violência Contra a Mulher ${ }^{3}$. Esta foi instituída, em 2011, por meio da Secretaria Nacional de Políticas para as Mulheres visando desenvolver estratégias efetivas para o empoderamento feminino, garantia de seus direitos humanos, responsabilização dos agressores e promoção da assistência qualificada às mulheres em situação de violência, fazendo-se cumprir ao que é preconizado pela Política Nacional de Enfrentamento à Violência contra as Mulheres ${ }^{8}$.

Na Rede de Enfrentamento à Violência Contra as Mulheres, se insere a Rede de Atendimento, que é composta por uma gama de serviços especializados e não especializados, incluindo a Estratégia de Saúde da Família (ESF) e o Núcleo de Apoio à Saúde da Família (NASF) ${ }^{3}$. Nesse conjunto, a assistência se faz por meio de ações como a identificação e encaminhamento adequado dos casos de violência contra a mulher, priorizando a integralidade e a humanização no atendimento ${ }^{3,8}$. A atuação dos profissionais desses serviços, enquanto integrantes da referida Rede, compreende, sobretudo, a identificação precoce dos sinais e sintomas de violência, realização do acolhimento, atendimento, notificação dos casos e encaminhamento para outros serviços de atenção especializados e de proteção social ${ }^{9}$.

É importante ressaltar que, apesar dos significativos avanços legais no enfrentamento aos casos de violência, os números revelam persistência da vitimização feminina. Dados do Mapa da Violência de 2015 demonstram uma taxa de 49,2\% de reincidência das mulheres aos serviços de saúde por danos decorrentes da violência doméstica, o que sugere uma baixa resolutividade da assistência ofertada nos serviços de saúde, sobretudo, na APS, considerando-se o nível de atenção ordenador do cuidado ${ }^{10,11}$. Pesquisa internacional também aponta que mulheres, que buscaram serviços de emergência, estão mais propensas a experienciar novas situações de abuso nos meses subsequentes e necessitarem de novo atendimento no serviço ${ }^{12}$.

Em meio a esse contexto, considera-se imprescindível conhecer quais são as condições que têm interferido no cuidado à saúde da mulher em situação de violência conjugal no âmbito da Estratégia de Saúde da Família (ESF), uma vez que identificar essas condições podem direcionar estratégias para prevenção de agravos decorrentes do fenômeno, inclusive o feminicídio. Acredita-se que, a partir desse conhecimento conseguirá compreender o fluxo utilizado pelos profissionais da Rede de Atendimento, no cenário da saúde, bem como o seu preparo na condução dos casos. Dessa forma, o estudo tem como objetivo conhecer as condições que interferem no cuidado à mulher em situação de violência conjugal.

\section{MÉTODO}

Trata-se de uma pesquisa qualitativa ancorada na Teoria Fundamentada nos Dados (TFD). A escolha pelo método se deu porque busca compreender as formas como os seres sociais vivenciam suas experiências a partir da valorização das subjetividades $^{13}$. A lista de verificação intitulada Consolidated Criteria for Reporting Qualitative Research (COREQ) foi utilizada para delinear o desenho do estudo e orientar as etapas da pesquisa, sendo prioridade das autoras atender integralmente aos critérios nela apresentados.

O estudo integra um projeto central contemplado por edital do Programa Pesquisa para o SUS: Gestão Compartilhada em Saúde, intitulado "Enfrentamento da violência conjugal no âmbito do Sistema Único de Saúde: tecnologia social envolvendo mulheres, homens e profissionais da atenção básica".

Para a coleta dos dados, estabeleceu-se enquanto cenário do estudo 22 Unidades de Saúde da Família (USF) situadas em um mesmo distrito sanitário de uma cidade do Nordeste brasileiro. Ao menos, uma representante de cada USF foi intencionalmente convidada para compor a amostragem teórica do estudo. As profissionais de saúde atuantes nesse cenário, que integram a equipe mínima da ESF (enfermeiras, médicas e dentistas) e equipe do NASF (psicóloga e assistente social), foram contactadas por via telefônica, para compor o estudo através de entrevista individual. Na ligação, compartilhou-se o tema e objetivo da pesquisa e foi 
agendado local e momento para realização da entrevista. Vale salientar que o estudo possui parceria com a Secretaria Municipal de Saúde do Estado e coordenação da Área Técnica em Saúde da Mulher, que cederam os contatos telefônicos e informaram previamente os funcionários sobre o projeto.

Foi adotado como critério para inclusão na pesquisa possuir atuação na ESF por no mínimo seis meses, que ao entendimento das pesquisadoras, é o tempo mínimo necessário para que haja uma vinculação com as usuárias do serviço e que essas possam compartilhar de suas histórias de vida. Excluiu-se aquelas profissionais que estavam afastadas do cargo por atestado médico ou não compareceram no momento da entrevista, por três vezes consecutivas sem justificativa plausível, totalizaram 31 profissionais. Esse quantitativo de participantes não foi previamente delimitado pelas pesquisadoras, sendo prioridade a apuração do conteúdo e consistência dos dados até que fosse constatada a saturação teórica, marcada pela ocorrência de repetições e ausência de novos dados ${ }^{14}$.

Essa etapa de coleta, abarcando dois grupos amostrais, ocorreu entre fevereiro e dezembro de 2019 e foi realizada em sala privativa na própria Unidade de Saúde de atuação das profissionais. Essas entrevistas foram conduzidas por pósgraduadas com experiência na realização de entrevistas. $O$ primeiro grupo amostral foi formado por 26 integrantes das equipes mínimas da USF e o segundo composto por cinco profissionais do NASF. Para nortear essas entrevistas, utilizou-se um formulário semiestruturado contendo questões fechadas, com vistas a traçar um breve perfil das profissionais, e abertas possibilitando que falassem livremente sobre suas vivências, norteadas pela seguinte provocação: "Fale-me sobre as facilidades e entraves para cuidar de mulheres em situação de violência conjugal".

Atendendo ao proposto pela TFD, as entrevistas gravadas em smartphone e, em seguida, transcritas na íntegra em um documento do Microsoft Word, sendo organizadas e categorizadas, à medida que foram sendo realizadas com as participantes. Salienta-se que as transcrições foram executadas por graduandas que receberam capacitação prévia e validadas pelas pesquisadoras responsáveis que estavam presentes nas entrevistas, bem como pelas participantes do estudo. De maneira a facilitar a organização e codificação dos dados, nessa etapa do trabalho, foi utilizada a ferramenta computacional NVIVO 10. Em posse das informações oriundas do primeiro grupo amostral, as pesquisadoras realizaram análise minuciosa, da qual emergiu a hipótese que as direcionou para o segundo grupo amostral, com quem foi realizada a mesma indagação.

Para a análise dos dados, as entrevistas, que tiveram duração média de 50 minutos, foram submetidas às etapas de codificação e análise previstas na TFD, sendo elas codificação aberta, axial e integração. Nessa última etapa, foram formuladas categorias analíticas a partir dos elementos do modelo paradigmático: condições, ações-interações e consequências/resultados, permitindo desvelar o fenômeno "Revelando que o cuidado à mulher para o enfrentamento à violência conjugal atrela-se a ações de identificação e intervenção do agravo condicionadas pelo interesse da gestão nos diferentes níveis". A validação desse fenômeno foi realizada por três pesquisadoras(es) com expertise no método e, também, por três entrevistadas do estudo.

Neste artigo, será apresentado apenas o componente que diz respeito às condições do fenômeno, encontrado nas narrativas de profissionais que atuam na ESF e fazem referência aos elementos que interferem no cuidado à mulher em situação de violência conjugal. Esses elementos estão representados nas categorias: Entendendo a importância da atuação profissional organizada; Reconhecendo a necessidade de preparo profissional para enfrentamento da violência conjugal; Percebendo a essencialidade do fluxo de atendimento intersetorial.

No que se refere aos aspectos éticos, salienta-se que o presente estudo possui aprovação do Comitê de Ética em Pesquisa (CAAE: 88960217.6.0000.5531/Número do Parecer: 2.639.224/2018). Para além, no momento que precedeu a coleta, foi lido, individualmente, o Termo de Consentimento Livre e Esclarecido e obtida a anuência das participantes através da assinatura desse documento. Nele constam objetivo, riscos e benefícios da participação na investigação. Somado a isso, a partir dele é assegurado às colaboradoras o sigilo e anonimato, estando esse último relacionado à substituição dos seus nomes por um código que segue o exemplo: "E1, G1", sendo o numeral que sucede a letra "E" referente à ordem da entrevista e aquele acrescido ao "G", ao grupo amostral que o participante integra.

\section{RESULTADOS}

Participaram do estudo 31 profissionais de saúde que atuam nas USF's e no NASF da ESF, sendo duas assistentes sociais, três psicólogas, quatro dentistas, cinco médicas e 17 enfermeiras. A faixa etária ficou entre 28 a 65 anos, enquanto que o tempo de atuação desses profissionais na ESF varia entre um e 26 anos. Quanto a capacitação profissional, 25 deles têm pós-graduação lato sensu sendo a maior concentração na área de saúde pública $(n=10)$ e saúde da família $(n=4)$.

Com base nas narrativas das entrevistadas, o cuidado à mulher em situação de violência conjugal no âmbito da ESF sofre influência do interesse da gestão, nos níveis locais, municipais, estaduais e federais, de modo a favorecer ou comprometer a organização do processo de trabalho, o preparo profissional e o fluxo de atendimento intersetorial. Essas categorias encontramse apresentadas, a seguir:

\section{Entendendo a importância da atuação profissional organizada}

Ao tecer crítica à lógica da produtividade, que limita o tempo destinado ao atendimento, os profissionais assinalam a importância da escuta qualificada e construção de vínculos entre profissional-usuária. Nesse sentido, sinalizam que a atuação profissional organizada favorecerá o cuidado à mulher que vivencia um contexto de violência conjugal.

Por ser um Programa de Saúde da Família, tenho uma vinculação com a mulher, conheço a história dela, mas 
o tempo de consulta não permite fazer uma escuta qualificada. Existe uma exigência por produção e, com isso, as situações de violência passam despercebidas. Isso teria que mudar! (E15, G1)

Tenho um tempo pré-estipulado para ficar com cada paciente. Não consigo chegar a assuntos tão profundos quanto violência no relacionamento íntimo nesse tempo. (E5, G1)

É a partir do vínculo que a gente consegue estabelecer confiança para a mulher revelar algumas situações, como violência conjugal. Nós (do NASF) dispomos de mais tempo para o atendimento, mas fica difícil para os profissionais da unidade, com o tempo limitado para atender as usuárias (E1, G2)

\section{Reconhecendo a necessidade de preparo profissional para enfrentamento da violência conjugal}

A capacitação profissional também foi sinalizada nas falas das participantes como condição que interfere no cuidado à mulher que experiencia a violência conjugal. Diante o reconhecimento da dificuldade de abordar e lidar com essa questão, entendem ser imperativa a capacitação profissional, a ser promovida pela gestão nos diferentes níveis, o que perpassa pela incorporação da temática na formação acadêmica e a promoção de espaços institucionais para atualizações em serviço.

Me senti impotente porque ela não quis denunciar, não se sentia preparada para sair do relacionamento e eu não sabia o que fazer para ajudá-la. $A$ fragilidade com o tema da violência vem desde a graduação porque não fomos capacitados para abordar esse assunto. [...] precisávamos receber mais capacitações nessa temática tão delicada (E20, G1)

A gestão precisa qualificar os profissionais porque muitos sequer sabem como abordar a mulher se ela sofre violência. Já recebemos diversos treinamentos e capacitações, tanto da secretária municipal como do Ministério da Saúde, mas isso nem sempre alcança todos na unidade. Percebo que os profissionais da equipe mínima não se sentem aptos para notificar e esperam por nós, psicólogas e assistentes sociais. (E3, G2)

A maioria dos profissionais da equipe mínima não está apta para investigar situações de violência conjugal, então, eles acabam não abordando essa temática na sua rotina de atendimento às mulheres. (E4, G2)

\section{Percebendo a essencialidade do fluxo de atendimento intersetorial}

O fluxo de atendimento intersetorial foi outra condição, apontada no estudo, como influenciadora para o cuidado ou não da mulher em situação de violência conjugal. Ao perceber a fragilidade da rede de atenção, os profissionais sinalizam para a relevância do funcionamento adequado desse fluxo por meio da articulação entre os serviços, que pode ser direcionada pela gestão em diferentes instâncias.

Na unidade onde eu trabalho não existe um fluxo de encaminhamento das mulheres em situação de violência conjugal. Se soubesse quais são os primeiros passos que devo dar, acredito que ajudaria muito a mulher a buscar apoio em locais certos. (E7, G1)

Precisamos de um fluxo organizado, mas a rede é frágil e, muitas vezes, dependemos da boa vontade do colega para encaminhar a mulher. (E4, G2)

Esbarramos no fluxo de atendimento à mulher em situação de violência que está fragmentado. Então, ela precisa ficar indo há vários lugares serviços como Unidade de Pronto Atendimento, Delegacia, Promotoria. [...] não vejo comunicação da atenção básica com esses serviços. (E15, G1)

\section{DISCUSSÃO}

Os relatos das profissionais de saúde mostram que a organização do processo de trabalho no âmbito da ESF se apresenta enquanto influenciador positivo e/ou negativo no cuidado à mulher em situação de violência conjugal, visto que promoverá ou não a construção de vínculos entre profissionais e usuárias. As narrativas revelam que as profissionais reconhecem esses vínculos enquanto uma condição essencial para o estabelecimento de uma relação de confiança que permita o desvelar da vivência de fenômenos de difícil verbalização, como a violência no cenário da conjugalidade.

Todavia, é importante salientar que esse processo de vinculação pode ser oportunizado em quaisquer momentos de interação com as mulheres, não se restringindo às consultas formais como, por exemplo, atividades em grupo, visitas domiciliares, aplicação de vacinas, realização de curativos etc. Nessa conjuntura, a ESF, que representa uma estratégia organizada através do cadastramento de famílias por equipe de referência, favorece a continuidade do cuidado pelos profissionais e, assim, fortalece a relação de proximidade. Corroborando, estudo qualitativo realizado com trabalhadoras(es) atuantes em ESF's do sudeste brasileiro revela que, a partir da constituição de vínculo, a identificação dos casos de violência, bem como o desenvolvimento do processo de cuidado pode ocorrer nos diversos espaços de socialização da comunidade adscrita, a exemplo de escolas, centros comunitários, igrejas dentre outros ${ }^{15}$

É importante referir que a violência conjugal representa uma problemática que agrega elementos suficientes para fazêla assumir o status de prioridade na dinâmica do trabalho da ESF, haja vista as inúmeras repercussões para a vida e saúde das mulheres além do risco eminente para o feminicídio, não 
justificando, portanto, a limitação de tempo destinado a essa assistência. Pelo contrário, o tempo de escuta do profissional da ESF junto a essas mulheres deveria ser o necessário para se estabelecer vínculos suficientes a fim de proporcionar a confiança destas e estimulando, assim, os relatos da violência conjugal vivenciada. Portanto, essa restrição de tempo é apontada como limitação para o cuidado às mulheres, em diferentes estudos ${ }^{16,17}$.

Para driblar essa dificuldade, pesquisa multimétodos conduzida em uma comunidade nepalesa sugere que a atuação das equipes com a comunidade pode favorecer mudanças na realidade local e influenciar positivamente as atitudes e práticas individuais em torno da violência contra a mulher. Para tanto, o estudo recomenda envolver a temática em diferentes cenários, como programas de rádios comunitários, encontros grupais, teatro de rua e mensagens de parceria com líderes locais, otimizando o tempo e ampliando o alcance das ações de enfrentamento ${ }^{18}$. Assim, as profissionais de saúde precisam assumir a responsabilização pelo cuidado e, dentro de suas possibilidades, criar estratégias para assegurar uma escuta qualificada e atender às necessidades das usuárias, com vistas ao enfrentamento do agravo.

Outra condição revelada nos discursos enquanto influenciador na prestação do cuidado, guarda relação com a falta de capacitação profissional necessária para realizar o atendimento às mulheres que vivenciam a violência, elemento evidenciado pelo sentimento de impotência pela não resolutividade dos casos. Frente a complexidade do fenômeno da violência, muitos profissionais que integram as equipes da Atenção Primária à Saúde (APS) se sentem despreparados para o atendimento à mulher. Tal dificuldade perpassa, muitas vezes, pela limitação durante a abordagem da temática, visto que na concepção social, trata-se de um problema de foro íntimo que deve ser resolvido no âmbito doméstico ou nas instâncias jurídicas. Estudos brasileiros e outro palestino relacionam a precariedade da formação do profissional de saúde no campo da violência com a necessidade de uma formação que aborde a temática da violência de forma articulada com o cuidado à saúde biopsicoespiritual de mulheres que enfrentam o fenômeno ${ }^{19-21}$.

Mesmo quando os profissionais intervêm, aconselhando a mulher para a representação da violência junto à delegacia, é comum que elas não acatem o encaminhamento, gerando, muitas vezes, na profissional o sentimento de impotência, conforme relatado. Diante desse cenário, faz-se necessário que as profissionais busquem alternativas que suplantam o encaminhamento para a Delegacia Especializada de Atendimento à Mulher (DEAM), como a inserção da mulher em grupos educativos promovidos pela USF, o mapeamento de uma rede social de apoio que possa ser acionada como parceira nesse enfrentamento, podendo essa ser formada por familiares, amigos, líderes religiosos, entre outros sujeitos ${ }^{17}$. Acredita-se que tais espaços sejam essenciais para o processo de fortalecimento da mulher e elaboração de uma rede de apoio e proteção, elementos necessários para o enfrentamento da violência, considerando que a denúncia do abuso pode fragilizar ainda mais a vítima e deixá-la exposta a represália por parte do agressor ${ }^{22}$.

Vale destacar ainda a importância de as profissionais de saúde conhecerem os centros de apoio ou referências para atendimento à mulher em situação de violência. Esses espaços, em âmbito nacional ou internacional, se configuram como cenários estratégicos responsáveis em promover o acolhimento psicológico, social e jurídico; atendimentos especializados por equipe interdisciplinar; estabelecer articulação com outros serviços, organizações governamentais e não governamentais; além de contribuir para o fortalecimento das mulheres ${ }^{23-25}$.

No âmbito da ESF, a intervenção multiprofissional, principalmente aquela atrelada ao matriciamento, pode configurar-se também como suporte na atuação frente aos casos de violência. A discussão de casos identificados na comunidade com o NASF possibilita o trabalho conjunto com psicóloga, assistente social e terapeuta ocupacional, tendo como foco o cuidado à mulher inserida no contexto violento. As trocas e compartilhamentos podem ocorrer, por exemplo, durante as reuniões de equipe sendo inclusive os casos de violência, prioridade entre outras pautas. Pesquisas cubana e espanhola destacam a importância da atuação multiprofissional frente aos casos de violência conjugal no âmbito da APS. Esse trabalho conjunto amplia as possibilidades terapêuticas de cuidado à saúde física e mental, além de favorecer a proteção social às vítimas, tornando-as mais conscientes e responsáveis para as a tomada de decisões e, assim, ajudando-as a libertarem-se de condições abusivas ${ }^{26,27}$.

Tal partilha entre os profissionais pode também auxiliar a dirimir as inabilidades diante a formação deficitária e a carência de capacitações, conforme evidenciado nas narrativas. Essa não é uma realidade exclusiva do Brasil, estudo realizado com estudantes de enfermagem das universidades espanholas de Barcelona (Universidade de Barcelona e Universidade Autônoma de Barcelona), Tarragona (Universidade Rovira i Virgili) e Girona (Universidade de Girona) evidenciam que, durante seu processo formativo, esses discentes tiveram pouco ou nenhum contato com a temática da violência conjugal, apontando para a necessidade de mudanças no currículo formador com vistas ao desenvolvimento de competências e habilidades no manejo desses $\operatorname{casos}^{28}$.

Todavia, ainda que existam lacunas na formação profissional no que se trata da abordagem dessa temática, urge a necessidade dessas trabalhadoras buscarem de forma autônoma o conhecimento, por meio de documentos disponíveis gratuitamente nos meios digitais. A busca por arcabouço teórico para a construção do próprio aprendizado favorece o empoderamento das profissionais de saúde, o que as estimula a prestar um cuidado mais qualificado. Ressalta-se que além do conhecimento científico, aquele de cunho empírico é dominado por quem atua nas USFs e deve ser valorizado, pois é igualmente significativo, uma vez que abarca as particularidades que envolvem a realidade territorial, as experiências dos moradores e as próprias percepções adquiridas ao longo tempo de atuação profissional ${ }^{29}$. 
Necessário se faz salientar que eventuais danos causados às usuárias do serviço de saúde, no caso desse estudo, as mulheres em situação de violência conjugal, por parte dos profissionais de saúde são passíveis de responsabilidade nas esferas cível e penal. Tal situação, definida como imperícia, está contemplada nos códigos de éticas de diferentes profissões da saúde, a exemplo da Medicina e da Enfermagem ${ }^{30,31}$. Com isso, mais do que uma motivação, buscar capacitar-se para a atuação frente a fenômenos emergentes da comunidade assistida, em particular a violência conjugal, configura-se enquanto uma obrigação legal do exercício profissional.

Apesar de terem ainda uma visão restrita sobre a construção do conhecimento, com dificuldades de perceberem-se enquanto agentes na busca do saber, as entrevistadas compreendem a necessidade de serem melhores capacitadas para atuar frente aos casos. Além do que os relatos também tornam perceptíveis que as capacitações não vêm sendo ofertadas de forma horizontal para todas as profissionais, uma vez que ao contrário do que acontece com aqueles que atuam no NASF, a equipe mínima das USF's nem sempre é contemplada com tais atividades, o que pode, associado às especificidades das profissões, justificar o fato das narrativas apontarem para indicação das mulheres às psicólogas e assistentes sociais.

Outra situação que coloca em relevo a ESF é a sua organização. Apesar dos nossos dados revelarem uma média de nove anos de atuação na ESF, essa não representa uma realidade nacional. Estudo realizado com 811 profissionais de saúde vinculadas(os) a equipes de ESF de um município de Minas Gerais evidenciou que a média de tempo de permanência dos profissionais foi de 20 meses, sendo que mais de $48 \%$ das profissionais permanecem por tempo inferior a 12 meses $^{32}$.

A alta rotatividade de profissionais na equipe pode ser um entrave para o aprimoramento da assistência prestada. A entrada e saída de profissionais necessariamente ocasiona a ruptura dos processos e prejudica a qualidade dos atendimentos, conforme assinala estudo espanhol ao revelar que a alternância de profissionais afeta a coesão das equipes e compromete a capacidade destes se envolverem e estabelecerem relações de confiança com colegas e a comunidade ${ }^{33}$. Diante dessa realidade, táticas de gestão precisam ser pensadas por diferentes esferas de governo a fim de suprimir tal alternância nos postos de trabalho. Isso porque, os esforços destinados a prevenção e enfrentamento da violência contra a mulher, temática sensível e que precisa de relações estreitas de proximidade para ser revelada, sofre particular impacto com a alta rotatividade de trabalhadores na ESF.

Vale salientar que o interesse da gestão em capacitações por si só não garante necessariamente as mudanças esperadas, sendo importante também que as profissionais reflitam criticamente sobre a temática e assumam compromissos diante de sua atuação frente ao agravo. Nessa seara, a utilização de metodologias ativas que contemplem os casos das usuárias e a realidade local pode se configurar como ferramenta, pois tornam mais palpável e exequível os planos de cuidado às mulheres que vivenciam violência. Os benefícios advindos pela ampliação do conhecimento e aquisição de novos saberes devem ser coletivos, podendo ser desfrutados pelas mulheres que recebem o cuidado qualificado, tendo suas demandas atendidas; a sua família, que indiretamente pode sofrer pelas agressões; e os profissionais que, além da sensação de bem-estar, poderão usufruir do aprendizado em situações posteriores.

Os relatos expressaram a valorização da existência do fluxo de atendimento intersetorial pelas profissionais, reconhecendo que a sua inexistência impacta negativamente no cuidado prestado. Essa realidade pode guardar relação com o fato do despreparo profissional, anteriormente pontuado, quando as entrevistadas desse estudo desconhecem os serviços e as rotas de encaminhamento para mulheres em situação de violência conjugal. Situação similar foi relatada em estudo realizado na África do Sul, no qual as enfermeiras entrevistadas só reconheciam a assistência social como possibilidade de referência e mesmo assim esse encaminhamento é vedado ao profissional médico ${ }^{34}$. Para superar as dificuldades no manejo dos casos de violência conjugal contra a mulher, Portugal conta, desde 2012, com uma ferramenta técnica detalhada para orientar na identificação, monitoramento e encaminhamento das situações de violência identificadas nos serviços ${ }^{35}$.

As profissionais entrevistadas neste estudo verbalizaram haver uma carência de documentos institucionais que direcionem o caminho a ser percorrido pela usuária na rede de atenção. Vale destacar que na ausência desses materiais faz-se necessário que as(os) trabalhadoras(es) que prestam atendimento compartilhem com a gestão a responsabilidade de promover a estruturação da rede de assistência. Para tanto, aquelas(es) que atuam na USF, detentoras(es) do conhecimento sobre as especificidades da população que assiste, podem ser agente mobilizador do contato com outros serviços, o que poderá favorecer a construção de uma rede. Ratificando tais achados, estudo internacional revela que a eficácia da rede de atenção as mulheres em situação de violência guardam relação com a organização de fluxos estabelecidos, com representação de instituições distintas como jurídico policial, assistência social e saúde, sendo fundamental que estas possuam autonomia para decidir, planejar e executar ações para prevenção e enfrentamento do fenômeno ${ }^{36}$.

Ainda que essa rede se constituísse informalmente no início, a partir do networking entre os profissionais que buscariam conexões, compartilhando informações, a posteriori seria possível que, com o apoio da gestão, elas fossem organizadas, estruturadas e fortalecidas. Assim, diferentes serviços podem ser gradualmente integrados e, consequentemente, o fluxo de atendimento às mulheres em situação de violência ser construído. Pesquisas de cunho internacional apontam experiências exitosas na identificação e atuação de casos de violência conjugal na APS a partir de uma organização pautada na articulação com a rede de atenção e proteção social, grupos de pesquisa atuantes na prevenção e responsabilização social com as mulheres e espaços de apoio terapêutico para saúde mental ${ }^{26,27}$. 
Um mecanismo facilitador para comunicação entre os serviços que compõem a rede é o sistema de referência e contrarreferência. Esses dispositivos viabilizam o trânsito das usuárias nos diversos níveis de atenção, entretanto ainda é realizado timidamente pelos profissionais de saúde. Essa subutilização desse sistema pode justificar a ausência desses termos no discurso das participantes, além do que, pode-se sugerir que a falta de capacitação dessas profissionais na temática em estudo, como visto acima, possa ser uma das causas da subutilização do sistema de referência e contrarreferência. No entanto, por respaldarem trabalhadoras e usuárias quanto à assistência precisam ter sua aplicabilidade garantida.

\section{CONCLUSÃO E IMPLICAÇÕES PARA A PRÁTICA}

O estudo mostra que o cuidado à mulher em situação de violência conjugal pode estar limitado pela logística de organização dos serviços de saúde, a qual restringe o tempo da consulta, e pelo preparo profissional desde a graduação para atuar frente a esses casos. Soma-se a isso, o impacto da inexistência ou desconhecimento de um fluxo de atendimento intersetorial delineado, de maneira que pode fragilizar o encaminhamento da mulher vítima de violência e comprometer a sua inserção na Rede de Enfrentamento.

Nesse contexto, embora limite-se por não investigar os impactos dessas condições de cuidado na vida e saúde das mulheres com história de violência, o estudo avança por oferecer subsídios que podem orientar os gestores, nas esferas local, municipal, estadual e federal, no sentido de elaborar ações de identificação e enfrentamento do agravo, pautadas na coparticipação e corresponsabilização das trabalhadoras da Estratégia de Saúde da Família com fins em melhorias na assistência ofertada a esse público feminino.

Diante os relatos das profissionais que sinalizam para uma fragilidade no processo de formação acadêmica/profissional, o estudo aponta, ainda, a importância dos colegiados de instituições abordarem esse conteúdo nos componentes curriculares, a fim de trabalhar a temática da violência de forma ampla e melhor preparar graduandas para a realidade profissional. Ressalta-se, também, a importância da educação permanente em saúde, o que possibilita a atualização dos profissionais e favorece a sua aptidão para atuar auxiliando as mulheres no processo de enfrentamento da violência.

\section{FINANCIAMENTO}

O presente trabalho foi realizado com apoio da Coordenação de Aperfeiçoamento de Pessoal de Nível Superior - Brasil (CAPES) - Através do Programa de Doutorado Sanduíche no Exterior (PDSE)- Código de Financiamento 001", concedido a Jordana Brock Carneiro, à época, doutoranda do Programa de Pós-Graduação em Enfermagem e Saúde da Universidade Federal da Bahia, e financiado pela Fundação de Amparo à Pesquisa do Estado da Bahia (FAPESB), concedido a Profa. Dra.
Nadirlene Pereira Gomes, docente da Escola de Enfermagem da Universidade Federal da Bahia.

\section{CONTRIBUIÇÃO DOS AUTORES}

Desenho do estudo. Jordana Brock Carneiro. Nadirlene Pereira Gomes.

Coleta ou produção dos dados. Jordana Brock Carneiro. Nadirlene Pereira Gomes. Andrey Ferreira da Silva.

Análise de dados. Jordana Brock Carneiro. Nadirlene Pereira Gomes. Lilian Conceição Guimarães de Almeida. Natália Webler. Maria Deolinda Antunes Luz Lopes Dias Mauricio. Andrey Ferreira da Silva.

Interpretação dos resultados. Jordana Brock Carneiro. Nadirlene Pereira Gomes. Lilian Conceição Guimarães de Almeida. Cátia Maria Costa Romano.

Redação e revisão crítica do manuscrito. Jordana Brock Carneiro. Nadirlene Pereira Gomes. Lilian Conceição Guimarães de Almeida. Cátia Maria Costa Romano. Andrey Ferreira da Silva Natália Webler. Maria Deolinda Antunes Luz Lopes Dias Mauricio.

Aprovação da versão final do artigo. Jordana Brock Carneiro. Nadirlene Pereira Gomes. Lilian Conceição Guimarães de Almeida. Cátia Maria Costa Romano. Andrey Ferreira da Silva. Natália Webler. Maria Deolinda Antunes Luz Lopes Dias Mauricio.

Responsabilidade por todos os aspectos do conteúdo e a integridade do artigo publicado. Jordana Brock Carneiro. Nadirlene Pereira Gomes. Lilian Conceição Guimarães de Almeida. Cátia Maria Costa Romano. Andrey Ferreira da Silva. Natália Webler. Maria Deolinda Antunes Luz Lopes Dias Mauricio.

\section{EDITOR ASSOCIADO}

Cristina Rosa Baixinho (D)

\section{EDITOR CIENTÍFICO}

Ivone Evangelista Cabral (1)

\section{REFERÊNCIAS}

1. United Nations Entity for Gender Equality and the Empowerment of Women. Progress of the world's women 2019-2020: families in a changing world [Internet]. New York: UN Women; 2019 [citado 2021 jan 14]. Disponível em: https://www.unwomen.org/-/media/headquarters/ attachments/sections/library/publications/2019/progress-of-the-worldswomen-2019-2020-en.pdf?la=en\&vs=3512

2. Santos JDLB, Santos CVM. Considerações sobre a Rede de Enfrentamento à Violência Contra as Mulheres. Rev. Contexto Saude. 2020;20(40):13948. http://dx.doi.org/10.21527/2176-7114.2020.40.139-148.

3. Secretaria Nacional de Enfrentamento à Violência contra as Mulheres (BR), Secretaria de Políticas para as Mulheres. Rede de Enfrentamento à Violência contra as Mulheres [Internet]. Brasília; 2011 [citado 2021 jun 7]. Disponível em: https://www12.senado.leg.br/institucional/omv/ entenda-a-violencia/pdfs/rede-de-enfrentamento-a-violencia-contraas-mulheres

4. Fórum Brasileiro de Segurança Pública (BR). Anuário Brasileiro de Segurança Pública [Internet]. Brasília; 2019 [citado 2021 jan 14]. Disponíve em: http://www.forumseguranca.org.br/wp-content/uploads/2019/09/ Anuario-2019-FINAL-v3.pdf 
5. Ministério da Mulher, da Família e dos Direitos Humanos (BR). Painel de dados da ouvidoria nacional de direitos humanos: análise por perfil da vítima [Internet]. Brasília; 2021 [citado 2021 jun 7]. Disponível em: https://www.gov.br/mdh/pt-br/ondh/paineldedadosdaondh/dadosatuais-2021

6. Carneiro JB, Gomes NP, Campos LM, Silva AF, Cunha KS, Costa DMS. Understanding marital violence: a study in Grounded Theory. Rev Lat Am Enfermagem. 2019;27:e3185. http://dx.doi.org/10.1590/15188345.3116.3185.

7. Kozubik M, van Dijk JP, Rac I. health risks related to domestic violence against roma women. Int J Environ Res Public Health. 2020;17(19):6992. http://dx.doi.org/10.3390/ijerph17196992.

8. Secretaria Nacional de Enfrentamento à Violência contra as Mulheres (BR), Secretaria de Políticas para as Mulheres. Política Nacional de Enfrentamento à Violência Contra as Mulheres [Internet]. Brasília; 2011 [citado 2021 jan 14]. Disponível em: https://www12.senado.leg. br/institucional/omv/entenda-a-violencia/pdfs/politica-nacional-deenfrentamento-a-violencia-contra-as-mulheres

9. Ministério da Saúde (BR), Instituto Sírio-Libanês de Ensino e Pesquisa. Protocolos da atenção básica: saúde das mulheres [Internet]. Brasília; 2016 [citado 2021 jan 14]. Disponível em: http://bvsms.saude.gov.br/ bvs/publicacoes/protocolos_atencao_basica_saude_mulheres.pdf

10. Waiselfisz JJ. Mapa da violência 2015: homicídio de mulheres no Brasil [Internet. Brasília;2015 [citado 2021 jan 14]. Disponível em: http://www. onumulheres.org.br/wp-content/uploads/2016/04/MapaViolencia_2015_ mulheres.pdf

11. Portaria $\mathrm{n}^{\circ}$ 2.436, de 21 de setembro de 2017 (BR). Aprova a Política Nacional de Atenção Básica, estabelecendo a revisão de diretrizes para a organização da Atenção Básica, no âmbito do Sistema Único de Saúde (SUS). Diário Oficial da União, Brasília (DF), 22 set 2017: Seção 1:68.

12. Ahmad I, Ali PA, Rehman S, Talpur A, Dhingra K. Intimate partner violence screening in emergency department: a rapid review of the literature. $J$ Clin Nurs. 2017;26(21-22):3271-85. http://dx.doi.org/10.1111/jocn.13706.

13. Corbin J, Strauss A. Basics of qualitative research: techniques and procedures for developing Grounded Theory. California: SAGE; 2015.

14. Santos JLG, Cunha KS, Adamy EK, Backes MTS, Leite JL, Sousa FGM. Data analysis: comparison between the different methodological perspectives of the Grounded Theory. Rev Esc Enferm USP. 2018;52:e03303. http:// dx.doi.org/10.1590/s1980-220x2017021803303.

15. Vieira EM, Hasse M. Perceptions of professionals in an intersectorial network about the assistance of women in situation of violence. Interface. 2017;21(60):52-62. http://dx. doi.org/10.1590/1807-57622015.0357.

16. Walton LM, Aerts F, Burkhart H, Terry T. Intimate Partner Violence Screening and Implications for Health Care Providers. Online J Health Ethics. 2015;11(1). http://dx.doi.org/10.18785/ojhe.1101.05.

17. Matos M, Gonçalves M. Sleep and women intimate partner victimization: prevalence, effects and good practices in health care settings. Sleep Sci. 2019;12(1):35-42. http://dx.doi.org/10.5935/1984-0063.20190057.

18. Clark CJ, Shrestha B, Ferguson G, Shrestha PN, Calvert C, Gupta J et al. Impact of the Change Starts at Home Trial on Women's experience of intimate partner violence in Nepal. SSM Popul Health. 2020;10:100530. http://dx.doi.org/10.1016/j.ssmph.2019.100530.

19. Nóbrega VK, Pessoa Jr JM, Nascimento EG, Miranda FA. Resignation, violence and filing complaint: social representations of the male aggressor from the perspective of the female victim of aggression. Cienc Saúde Colet. 2019;24(7):2659-66. http://dx.doi.org/10.1590/141381232018247.16342017.

20. Estrela FM, Gomes NP, Gusmão ME, Lírio JG, Couto TM, Cerqueira TP. Demographic or socio-demographic aspects of women in domestic violence situations: a descriptive study. Online Braz J Nurs. 2016;15(3):423-32. http://dx.doi.org/10.17665/1676-4285.20165380.

21. Baloushah S, Mohammadi N, Taghizadeh Z, Taha AA, Farnam F. A whole life of threats: a qualitative exploration of lived experiences of palestinian women suffering from intimate partner violence. Int J Womens Health 2019;11:547-54. http://dx.doi.org/10.2147/JJWH.S216952.

22. Cordeiro DCS. Por que algumas mulheres não denunciam seus agressores? CSOnline Rev Eletrônica Cienc Sociais. 2018;27:365-83. http://dx.doi.org/10.34019/1981-2140.2018.17512.

23. Mafioletti TM, Peres AM, Larocca LM, Fontoura MP. Violence against women: historical trajectory of a care program (Curitiba - 1997-2014) Rev Bras Enferm. 2018;71(6):2907-15. http://dx.doi.org/10.1590/00347167-2017-0583.

24. U.S. Department of Health \& Human Services (US), Office on Women's Health. Relationships and safety: domestic or intimate partner violence [Internet]. Washington; 2018 [citado 2021 jan 14]. Disponivel em: https:// www.womenshealth.gov/relationships-and-safety/domestic-violence

25. Associação Portuguesa de Apoio à Vítimas. Onde estamos: Rede Nacional de Gabinetes de Apoio à Vítima [Internet]. Lisboa: APAV; 2020 [citado 2021 jan 14]. Disponível em: https://apav.pt/apav_v3/index.php/ $\mathrm{pt} / \mathrm{gav} / \mathrm{onde}$-estao

26. Torralbas-Fernández A, Calcerrada-Gutiérrez M. Using primary care to address violence against women in intimate partner relationships: professional training needs. MEDICC Rev. 2016;18(4):38-41. http:// dx.doi.org/10.37757/MR2016.V18.N4.8.

27. Goicolea I, Mosquera P, Briones-Vozmediano E, Otero-García L, GarcíaQuinto M, Vives-Cases C. Primary health care attributes and responses to intimate partner violence in Spain. Gac Sanit. 2017;31(3):187-93 http://dx.doi.org/10.1016/j.gaceta.2016.11.012.

28. Rigol-Cuadra A, Galbany-Estragué P, Fuentes-Pumarola C, BurjalesMartí MD, Rodríguez-Martín D, Ballester-Ferrando D. Perception of nursing students about couples' violence: knowledge, beliefs and professional role. Rev Lat Am Enfermagem. 2015;23(3):527-34. http:// dx.doi.org/10.1590/0104-1169.0357.2584.

29. Halcomb E, Smyth E, McInnes S. Job satisfaction and career intentions of registered nurses in primary health care: an integrative review. BMC Fam Pract. 2018;19(1):136. http://dx.doi.org/10.1186/s12875-018-08191.

30. Resolução COFEN nº 0564/2017 (BR). Aprova o novo Código de Ética dos Profissionais de Enfermagem. Diário Oficial da União, Brasília (DF), 6 dez 2017: Seção 1:233.

31. Resolução CFM $n^{\circ} 2.217$, de 27 de setembro de 2018 modificada pelas Resoluções CFM no 2.222/2018 e 2.226/2019 (BR) Código de Ética Médica. Diário Oficial da União, Brasília (DF), 1 nov 2018.

32. Tonelli BQ, Leal AP, Tonelli WF, Veloso DC, Gonçalves DP, Tonelli SQ. Rotatividade de profissionais da Estratégia Saúde da Família no município de Montes Claros, Minas Gerais, Brasil. RFO UPF. 2018;23(2):180-5 http://dx.doi.org/10.5335/rfo.v23i2.8314.

33. Madrazo-Pérez M, Parás-Bravo P, Rayón-Valpuesta E, Blanco-Fraile, Palacios-Ceña. The impact of health human resources policies in primary care nursing: a qualitative study. Int J Environ Res Public Health. 2019;16(19):3653. http://dx.doi.org/10.3390/ijerph16193653.

34. Van der Wath A. Women exposed to intimate partner violence: a Foucauldian discourse analysis of South African emergency nurses' perceptions. Afr Health Sci. 2019;19(2):1849-57. http://dx.doi.org/10.4314/ ahs.v19i2.7.

35. Direção Geral de Saúde (PT), Ação de Saúde sobre Género, Violência e Ciclo de Vida. Violência interpessoal: abordagem, diagnóstico e intervenção nos serviços de saúde [Internet]. Portugal: DGS;2016 [citado 2021 jan 14]. Disponível em: https://www.dgs.pt/accao-de-saude-paracriancas-e-jovens-em-risco/ficheiros-externos/violencia_interpessoalpdf.aspx

36. Machado DF, Almeida MA, Dias A, Castanheira ER. Violence against women: what happens when the Women's Protection Police Station is closed? Cien Saude Colet. 2020;25(2):483-94. http://dx.doi. org/10.1590/1413-81232020252.14092018. 